\title{
Endocrine Therapy for Breast Cancer: A Model of Hormonal Manipulation
}

\author{
Simon J. Johnston · Kwok-Leung Cheung
}

Received: May 8, 2018 / Published online: July 10, 2018

(C) The Author(s) 2018

\section{ABSTRACT}

Oestrogen receptor (ER) is the driving transcription factor in $70 \%$ of breast cancer. Endocrine therapies targeting the ER represent one of the most successful anticancer strategies to date. In the clinic, novel targeted agents are now being exploited in combination with established endocrine therapies to maximise efficacy. However, clinicians must balance this gain against the risk to patients of increased side effects with combination therapies. This article provides a succinct outline of the principles of hormonal manipulation in breast cancer, alongside the key evidence that underpins current clinical practice. As the role of endocrine therapy in breast cancer continues to expand, the challenge is to interpret the data and select the optimal strategy for a given clinical scenario.

Keywords: Breast cancer; Combination therapy; Endocrine therapy; Hormone; Oestrogen receptor; Targeted therapy

Enhanced digital features To view enhanced digital features for this article go to https://doi.org/10.6084/ m9.figshare.6621512.

S. J. Johnston · K.-L. Cheung ( $\varangle)$

School of Medicine, University of Nottingham, Nottingham, UK

e-mail: Kwok_leung.Cheung@nottingham.ac.uk

\section{INTRODUCTION}

Oestrogen receptor (ER) is the driving transcription factor in around $70 \%$ of breast cancer [1]. Circulating oestrogen (17 $\beta$-estradiol, E2) binds to the ligand-binding domain of ER in breast cancer cells. The E2-ER complex then forms a dimer and binds to oestrogen response elements (EREs) on the DNA in the cell nucleus. This process activates a set of ER target genes which ultimately lead to cell cycle progression and tumour proliferation [2].

Endocrine therapy is the mainstay of treatment for patients with invasive breast carcinoma that is positive by immunohistochemistry for ER protein expression (ER+). The foundation stone for current practice was laid in the late nineteenth century by George Beatson, who found that removal of the ovaries (oophorectomy) improved outcomes for women with advanced breast cancer-even before the hormone, oestrogen, was discovered [3]. More than half a century later, the nuclear receptor ER itself was discovered, thereby providing a mechanism for the tissue specificity of oestrogen action [4].

Today, endocrine therapy such as tamoxifen and aromatase inhibitors represent one of the most effective targeted cancer therapies to reach the clinic. Other established targeted agents in $\mathrm{ER}+$ breast cancer are used largely in combination with endocrine therapy. These include monoclonal antibodies, tyrosine kinases, 
mammalian target of rapamycin (mTOR) inhibitors and cyclin-dependent kinase 4/6 (CDK4/ 6) inhibitors.

The clinical challenge now is to identify the right drug or therapy combination for the right patient for use at the right time. The aim is for individual patients to gain maximal benefit from endocrine therapy without incurring excessive toxicity.

This review is written as a resource for clinicians in training, scientists and Masters level students of oncology. This article is based on previously conducted studies, some of which were performed by the authors in full compliance with ethics guidelines. The article does not contain any studies with animals performed by either of the authors.

\section{TARGETING THE OESTROGEN RECEPTOR PATHWAY}

Hormonal manipulation of oestrogen signalling via $E R$ is achieved in a variety of ways in the treatment of breast cancer. The main strategies are oestrogen deprivation using aromatase inhibitors (AIs), and blocking the binding between oestrogen and ER using selective oestrogen receptor modulators (SERMs) or downregulators (SERDs). Hormonal manipulation may also be achieved with surgery and radiotherapy, although these modalities have largely been replaced by pharmacological strategies. Endocrine therapy is inherently less toxic than chemotherapy, which is not targeted on the ER. However, pharmacological strategies targeting an active ER have a range of adverse effects that can cause significant morbidity and even be life-threatening.

The choice of endocrine therapy in a given clinical context is a factor of age, menopausal status, co-morbidities and the drug toxicity profile. A balance between efficacy and tolerability (or toxicity) should be considered.

Tamoxifen is a SERM that competes with oestrogen for binding to the ligand binding domain of ER [5]. It is useful as a single agent for both pre- and postmenopausal patients [6].

In breast cancer tissue, tamoxifen-bound ER binds to the same genomic binding sites as oestrogen-bound ER. However, target genes are regulated in the opposite direction, with the downstream effect being reduced proliferation of tumour cells (ER antagonism) [5].

In other tissues, such as the endometrium, tamoxifen acts as a partial agonist. This is likely due to differential recruitment of co-activators and co-repressors to the ER transcription complex. In endometrial cells, tamoxifen-bound ER regulates ER target genes in the same direction as oestrogen-bound ER, leading to upregulated proliferation. Patients on tamoxifen for ER+ breast cancer increase their baseline risk of developing endometrial carcinoma by a factor of 2-3 [7].

Other potentially life-threatening adverse effects of tamoxifen include venous and arterial vascular events such as pulmonary embolism and stroke, whilst hot flushes impair quality of life and limit adherence to treatment [6]. Despite this, tamoxifen is generally well tolerated, with a side effect profile established over 40 years of use.

For postmenopausal women, endocrine therapy with third-generation aromatase inhibitors (AIs) such as anastrozole, letrozole and exemestane has now become the preferred choice over tamoxifen [8]. Aromatase inhibition decreases the peripheral conversion of androgens to oestrogen, thereby cutting off the tumour's fuel supply [9]. Anastrozole and letrozole are non-steroidal AIs. As a result of incomplete cross-resistance between steroidal and non-steroidal AIs, switching from anastrozole or letrozole to exemestane (or vice versa) may lead to change in disease response and/or toxicity [10].

Like tamoxifen, AIs are generally very well tolerated drugs with known toxicity profile. However, bone demineralisation and musculoskeletal side effects such as arthralgia cause significant treatment-related morbidity [11].

Fulvestrant is a selective ER downregulator. Like tamoxifen, it targets an active ER, but has a distinct mechanism of action [12]. Once bound, fulvestrant leads to ER degradation and loss of ER protein expression, which has been shown to be dose-dependent in a presurgical study (see Fig. 1) [13]. 


\section{Post-treatment Mean ER H-scores}

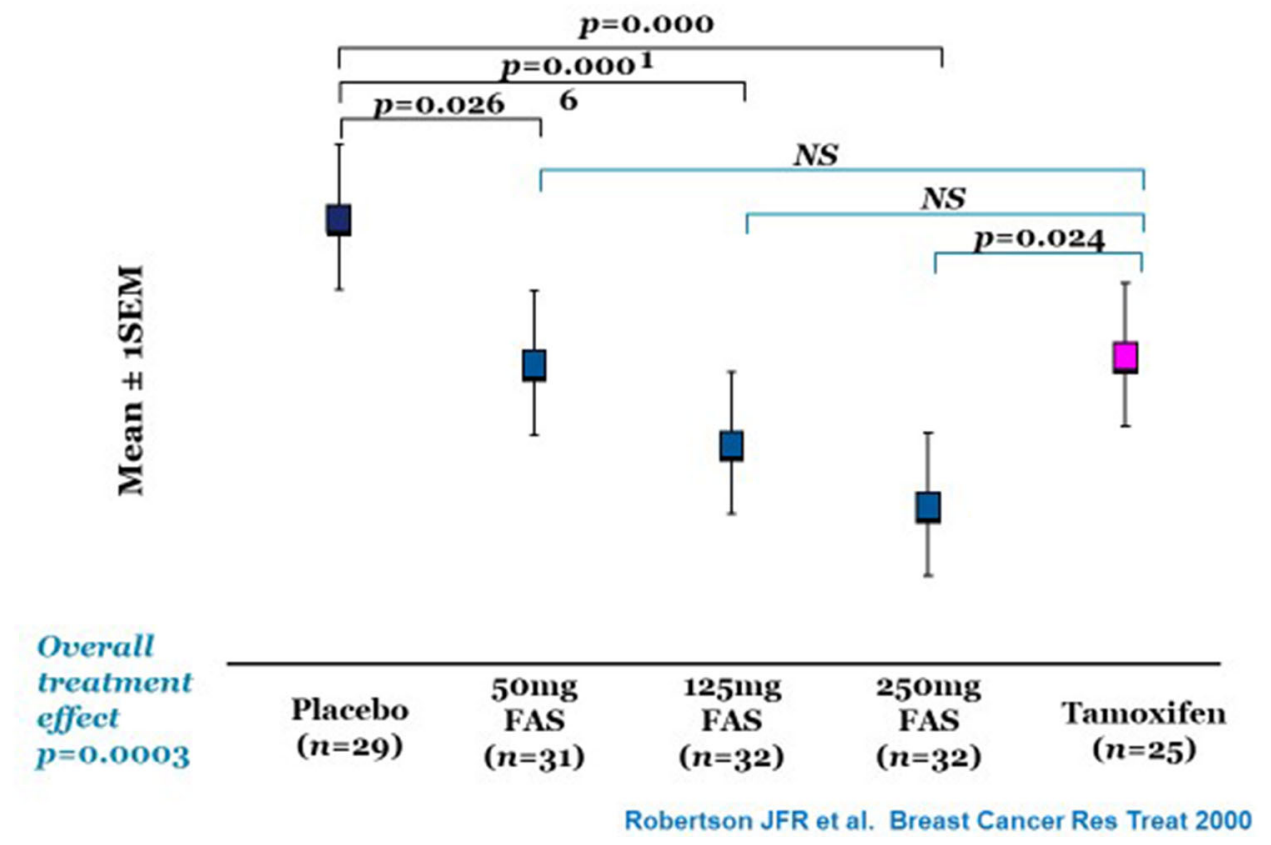

Fig. 1 Progressive reduction in post-treatment ER levels with increasing dose of fulvestrant. FAS Faslodex (fulvestrant), SEM standard error of the mean Reprinted from Ref. [13]. Copyright (2014), with permission from Elsevier

Fulvestrant has no known agonist activity, which avoids many of the pro-thrombotic and pro-proliferative side effects of tamoxifen. The side effect profile of fulvestrant also compares favourably with standard of care AIs, and causes fewer musculoskeletal side effects, as shown in the pivotal phase 3 trial comparing it with anastrozole (see Table 1) [14]. In contrast to AIs, which are known to be deleterious to bone mineral density, prospective clinical data with 18 months of follow-up indicate that fulvestrant does not increase bone turnover markers [15].

Newer targeted agents in current clinical use for ER+ breast cancer include mTOR inhibitors and CDK4/6 inhibitors. Although used in conjunction with endocrine therapies, both are purported to have specific activity in $\mathrm{ER}+$ disease.

Mammalian target of rapamycin is a serine/ threonine kinase downstream of phosphatidylinositol 3-kinase (PI3K)-Akt. Crosstalk between the PI3K-Akt-mTOR pathway and ER signalling is an evolving field. It is suggested that S6 kinase 1, a substrate of mTOR complex 1 (mTORC1), phosphorylates the activation function domain 1 of the ER leading to constitutive activity of ER [16]. By this mechanism, mTOR inhibition is claimed to have an indirect but specific effect on ER activity. Common side effects of mTOR inhibitors include rash, stomatitis, fatigue, diarrhoea and altered taste (dysgeusia).

The link between cyclin-dependent kinase $4 / 6$ inhibitors and ER activity is mechanistically less clear. Oncogenic pathways in ER+ breast cancer, e.g. PI3K-Akt-mTOR, ultimately converge on the cell cycle to drive cell proliferation. CDK4 and 6 form a cell cycle regulatory pathway with p16, cyclin $\mathrm{D}$ and the gatekeeper protein, retinoblastoma $(\mathrm{Rb})$. CDK4/6 inhibitors bind to CDK4 and CDK6, preventing phosphorylation of the $\mathrm{Rb}$ protein. This halts cell cycle progression and induces G1 cell cycle arrest 
Table 1 Tolerability of fulvestrant is similar to anastrozole but causes less musculoskeletal side effects. Modified with permission from Ref. [14]

\begin{tabular}{lccc}
\hline & \multicolumn{2}{l}{ Number of adverse events (\%) } & $\boldsymbol{p}$ value \\
\cline { 2 - 3 } & Fulvestrant & Anastrozole & 0.91 \\
Hot flushes & $89(21.0)$ & $87(20.6)$ & 0.53 \\
GI disturbance & $196(46.3)$ & $185(43.7)$ & 1.35 \\
Weight gain & $4(0.9)$ & $7(1.7)$ & 0.51 \\
Vaginitis & $11(2.6)$ & $8(1.9)$ & 0.68 \\
Thromboembolic disease & $15(3.5)$ & $17(4.0)$ & 0.0036 \\
Joint disorders & $23(5.4)$ & $45(10.6)$ & 0.062 \\
Urinary tract infection & $31(7.3)$ & $18(4.3)$ & \\
Withdrawn due to AE & $12(2.8)$ & $8(1.9)$ & \\
\hline
\end{tabular}

[17]. It is thought that resistance to endocrine therapy may occur because of cyclin D overexpression and retinoblastoma phosphorylation, making CDK4/6 inhibition an attractive strategy to improve outcomes for patients with hormone receptor-positive disease. Not surprisingly, the side effect profile of CDK4/6 inhibitors overlaps with other inhibitors of cell cycle progression, including chemotherapeutic agents. These include potentially life-threatening complications secondary to neutropenia. However, in contrast to that seen with chemotherapy, it is thought that such neutropenia is stem cell independent, less prolonged and less associated with febrile neutropenia [18].

\section{ADVANCED DISEASE}

In the advanced (non-curative) disease setting, endocrine therapy is used to control disease progression and improve survival. The principles of hormonal manipulation are best described in this setting because the tumour remains in situ, and the response to hormonal manipulation can usually be measured. Furthermore, since advanced disease carries a poor prognosis compared to early, non-metastatic disease, a shorter follow-up is required to reach primary endpoints in clinical trials. For these reasons, most drug development studies begin in this clinical context. It is generally only after both efficacy and tolerability have been shown to be satisfactory in the advanced setting that the potential use of new agents can be explored in the adjuvant setting.

Response to endocrine therapy in the advanced setting is assessed using established objective assessment criteria such as Response Evaluation Criteria in Solid Tumours (RECIST) and Union for International Cancer Control (UICC) (see Table 2).

Clinical benefit is a key principle in endocrine therapy decision-making in advanced $\mathrm{ER}+$ disease. Patients will be commenced on the best agent first. After 6 months of treatment, patients who have stable disease (SD) derive the same survival advantage as those who achieve an objective response (OR), compared to those who have progressive disease (PD) [19]. Patients with OR/SD at 6 months are therefore considered to have derived clinical benefit and can continue the same treatment until progression.

Patients with PD at 6 months can be considered as having intrinsic/de novo resistance to endocrine therapy. For these patients, the chance of deriving benefit to further endocrine manipulation is relatively low. This can, however, be optimised by selecting a sequential therapy with an alternative mechanism of action to minimise cross-resistance. 
Table 2 Assessment of responses (UICC)

Complete response (CR)

Partial response (PR)

Objective response (OR)

Stable disease (SD)

Progressive disease (PD)

Clinical benefit (CB)

$\mathrm{U} / \mathrm{A}$
No measurable disease

Reduction of at least $50 \%$ in maximal dimension

CR or PR

No change

Increase of at least $25 \%$ in maximal dimension, or new disease

$\mathrm{OR}+\mathrm{SD}$ at or beyond 6 months

Unassessable
Patients with CB at 6 months will eventually experience disease progression due to acquisition of secondary resistance mechanisms. Compared to de novo progressors, however, the chance of deriving benefit from sequential endocrine manipulation is relatively high.

Patients who present with advanced $\mathrm{ER}+$ disease can be new patients or pre-existing breast cancer patients whose disease has relapsed despite adjuvant therapy (a failure of adjuvant treatment). Initial therapy for advanced disease is based upon prior use of endocrine therapy in the adjuvant setting. The clinical focus of treatment in the non-curative setting is on quality of life. Endocrine therapy, being efficacious and generally well tolerated, is therefore the default treatment.

There are, however, certain circumstances in which chemotherapy is used first-line in advanced ER+ disease instead of endocrine therapy. Firstly, where there is imminently lifethreatening disease, e.g. extensive liver metastases or pulmonary lymphangitic spread with dyspnoea, chemotherapy can be used to provide more rapid disease control. Secondly, if endocrine resistance is likely (e.g. weak ER positivity, or no $\mathrm{CB}$ with prior endocrine therapy) single agent chemotherapy may be used.

Ovarian suppression in combination with endocrine therapy is a rational combination in premenopausal patients. Luteinising hormone releasing hormone analogue (LHRHa) therapy such as goserelin increases negative feedback on the pituitary, reducing the production of gonadotrophins.
In premenopausal women with advanced $\mathrm{ER}+$ disease, LHRHa therapy can be used firstline to suppress ovarian production of oestrogen. Goserelin is used in combination with tamoxifen, provided tamoxifen has not previously been used in the adjuvant setting. There is also evidence that LHRHa therapy in combination with aromatase inhibition works clinically and biochemically [20].

In postmenopausal women with advanced $\mathrm{ER}+$ disease, a third-generation AI (anastrozole, letrozole or exemestane) is superior to tamoxifen [21-23]. As an example, direct comparison with tamoxifen showed that anastrozole extends median time to progression by 4 months [24].

Targeted drugs that can be used in combination with endocrine therapy include CDK4/6 and mTOR inhibitors. These treatment options were incorporated into clinical guidelines following clinical trials data showing an additional 6 months of progression-free survival with mTOR inhibition (using everolimus plus AI), and 10 months with CDK4/6 inhibition (using palbociclib plus AI) $[16,25]$ compared to AI monotherapy.

Combination of an AI and CDK4/6 inhibitor has become standard first-line endocrine therapy in metastatic hormone receptor-positive breast cancer [26]. There are now three CDK4/6 inhibitors licensed in this setting: palbociclib [25], ribociclib [27] and abemaciclib [28]. The benefit over single agent AI therapy is not disputed but must be weighed against significant increase in toxicity when used in combination 
with CDK4/6 or mTOR inhibition, and patients must be selected accordingly.

Where combination therapy is not an option, fulvestrant $500 \mathrm{mg}$ may be used as sole therapy $[14,29]$. Trials of fulvestrant use in firstline advanced ER+ breast cancer included the FIRST (phase 2) and FALCON (phase 3) trials $[30,31]$. Whilst the phase 2 FIRST trial was not sufficiently powered to prove benefit, the phase 3 FALCON trial demonstrated superiority of fulvestrant over anastrozole (median PFS 16.6 versus 13.8 months) and led to fulvestrant being licensed for first-line use.

In second-line treatment, for patients who have not received prior CDK4/6 inhibitor and who have progressed on endocrine therapy, the preferred option is fulvestrant plus a CDK4/6 inhibitor [32]. An alternative option for secondline treatment following progression on $\mathrm{AI}$ is combination therapy with an mTOR inhibitor, everolimus [16].

Combination of fulvestrant with an AI has also been explored in clinical trials (see Table 3). This approach of combining ER antagonism (with SERM or SERD) with oestrogen deprivation (with AI) is known as "maximal endocrine therapy". However, additional benefit of the combination versus single agent therapy has been difficult to demonstrate, which may be due to prior adjuvant endocrine therapy usage (see Table 3). No difference is seen in both the SoFEA and FACT studies, in which most patients (around 70\%) had received prior endocrine therapy [33, 34]. In the later SWOG 0226 study, a smaller proportion of patients (around 40\%) had been treated with adjuvant endocrine therapy. The SWOG study found a significant improvement in PFS for patients who received the combination [35].

In patients with hormone receptor-positive disease that also overexpresses the HER2 receptor, endocrine therapy and HER2-targeted therapy can be used either sequentially or in combination. Comparison of these approaches was made in the Tandem study, which reported that trastuzumab plus anastrozole improves progression-free survival by a factor of 2 , albeit from a low baseline of 2.4 months, up to 4.8 months $(p=0.0016)$ [36]. However, this benefit came with significant cost in toxicity, with increase from $16 \%$ to $28 \%$ in grade 3 or 4 toxicities with use of the combination.

\section{ADJUVANT ENDOCRINE THERAPY}

In the curative setting, endocrine therapy is given as 'adjuvant' treatment for up to 10 years following definitive surgery, to reduce the risk of disease recurrence (relapse) for patients with $\mathrm{ER}+$ breast cancer. Additional chemotherapy is often used in patients with higher risk of relapse as determined by Nottingham Prognostic Index and other clinical risk stratification tools such as Adjuvant Online, Predict and Oncotype DX [37-39].

Choice of endocrine therapy drug in the adjuvant setting depends primarily on menopausal status. Tamoxifen as an adjuvant therapy works in pre- and postmenopausal women.

Premenopausal women, in whom levels of circulating oestrogen are higher as a result of

Table 3 Maximal endocrine therapy: effect of prior endocrine therapy

\begin{tabular}{llll}
\hline & SoFEA $^{\mathbf{a}}$ & FACT $^{\mathbf{b}}$ & ${\text { SWOG } \mathbf{~ 2 2 6}^{\mathbf{c}}}^{\mathbf{b}}$ \\
\hline $\begin{array}{l}\text { Design [F = fulvestrant (250 mg with loading dose); } \\
\text { A = anastrozole; E = exemestane] }\end{array}$ & $\mathrm{F}+\mathrm{A}$ vs F (vs E) & $\mathrm{F}+\mathrm{A}$ vs A & $\mathrm{F}+\mathrm{A}$ vs A \\
HP for PFS or TTP & 1.00 & 0.99 & 0.81 \\
Prior anti-oestrogen & $-70 \%$ & $-67 \%$ & $-40 \%$ \\
\hline
\end{tabular}

$H R$ hazard ratio, PFS progression-free survival, TTP time to progression

${ }^{\text {a }}$ Johnston et al. [33]

b Bergh et al. [34]

${ }^{c}$ Mehta et al. [35] 
endocrine production by the ovaries, are given tamoxifen to block the ER.

Premenopausal patients at higher risk of recurrence, in whom chemotherapy is indicated, are offered tamoxifen in combination with ovarian suppression [40]. The ZEBRA trial demonstrated that for women at higher risk who choose not to have chemotherapy, this approach can be an effective alternative. However, as a result of the date of the study, the comparison in ZEBRA was not made with modern chemotherapy regimes. More recently, the SOFT trial found that all women at higher risk of recurrence benefit further from subsequent ovarian suppression plus endocrine therapy following completion of chemotherapy [41].

In postmenopausal women, oestrogen is derived primarily from extra-gonadal sources. Inhibition of peripheral production of oestrogen via CYP19 (aromatase) using AIs has proved marginally more effective than blocking the ER with tamoxifen in postmenopausal women [11]. This conclusion has been reached on the basis of a collective view of all the adjuvant trials comparing the previous gold standard of 5 years of tamoxifen with AIs, with the latter approach demonstrating superiority both in terms of efficacy and tolerability [42]. The main concern of long-term (adjuvant) use of AIs is on bone mineral density (BMD). To prevent harm due to loss of $\mathrm{BMD}$, patients receiving AIs will have BMD checked at baseline, monitored regularly and managed if required using oral bisphosphonate medication.

Sequential and extended use of tamoxifen and AIs has been extensively explored (see Fig. 2).

In practice, menopausal status is often difficult to define, and a 'perimenopausal' status exists. This is further complicated by use of adjuvant chemotherapy that can cause menstrual cycles to cease, either temporarily or permanently. Pragmatic use of sequential tamoxifen and AI is often employed, backed by good evidence of equivalent effectiveness [43]. Strategies to define menopausal status may include measurement of circulating oestradiol and follicle-stimulating hormone levels.
Extended use of endocrine therapy has proved effective in further reducing rates of recurrence beyond the 5-year point, as demonstrated by pivotal studies such as MA17R, ATTOM and ATLAS [44-46]. In the clinic, this tends to be decided on a case-by-case basis depending on tolerability of endocrine therapy and risk of disease recurrence-especially distant metastases where the treatment intent will usually change to non-curative (i.e. palliative). The impact of prolonged endocrine therapy is most apparent from these studies when considering disease-free survival (DFS), which includes all events including local, regional and contralateral disease. However, there is a demonstrable but modest benefit on distant recurrence-free and overall survival, which continues beyond the 10 years of treatment.

\section{NEOADJUVANT ENDOCRINE THERAPY}

Chemotherapy can also be given prior to surgery, as a 'neoadjuvant' treatment with the aim of downstaging the primary tumour so that breast-conserving surgery can be performed (by wide local excision) instead of mastectomy.

Neoadjuvant therapy was originally proposed following work performed on a mouse model, which indicated that manipulation of the primary tumour leads to growth and dissemination of metastases [47]. The hypothesis was that neoadjuvant chemotherapy given to patients with primary breast cancer would treat undetectable metastases (micro-metastases) and thereby improve survival. However, the pivotal trial NSABP-B18 showed no difference in survival between chemotherapy given in the adjuvant and neoadjuvant settings [48]. This could be related to the fact that chemotherapy, as a result of its toxicity and immunosuppressive effects, could not be given during the perioperative period-the crucial period in terms of tumour manipulation.

In contrast, it is possible to give endocrine therapy in the perioperative period. While there is a concern that tamoxifen may further increase the risk of venous thromboembolism (VTE) during this period of high VTE risk, this 


\section{$x=$ Als Adjuvant Trial Designs}

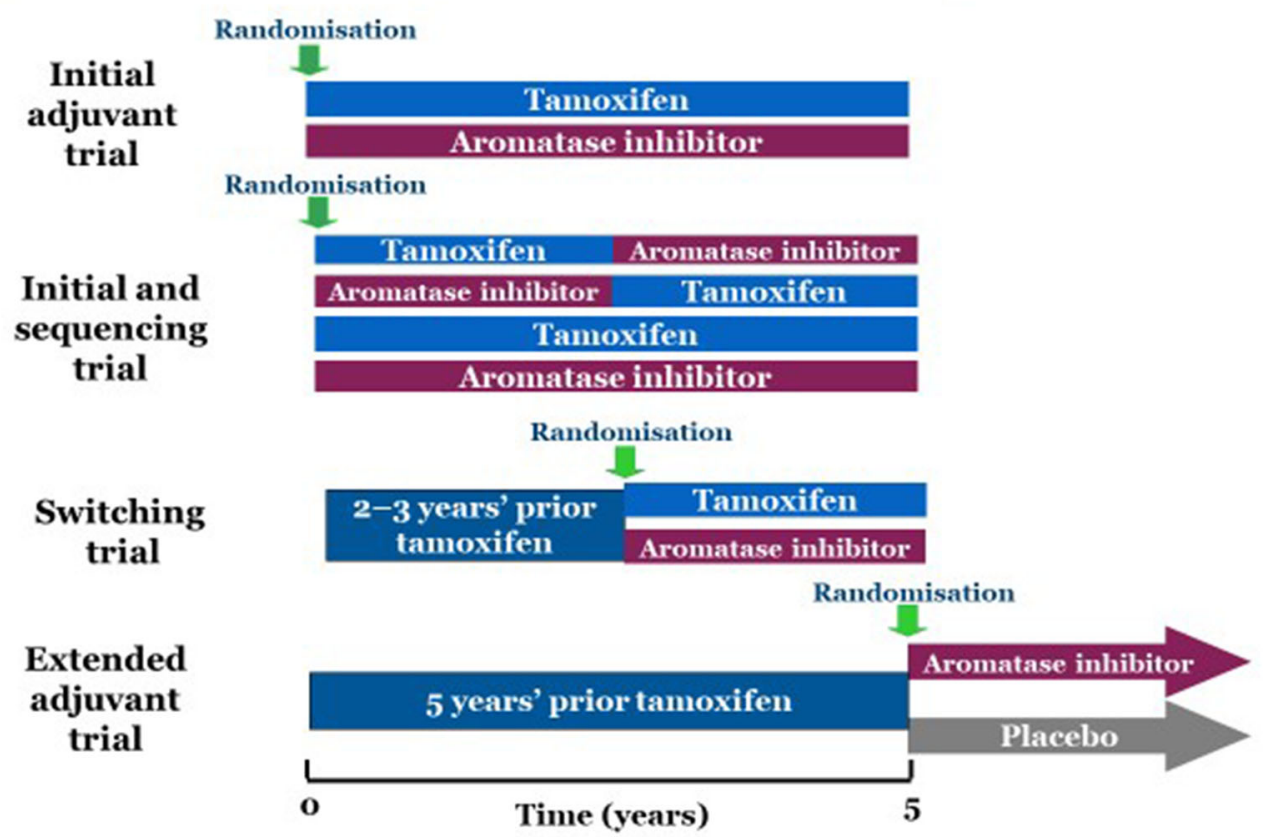

Fig. 2 Aromatase inhibitor adjuvant trial designs

would be avoided with the use of AIs or fulvestrant. In the neoadjuvant setting, the superiority of AIs over tamoxifen has been well established, and fulvestrant is also under investigation [49-52]. Both of these agents have a potential role in tumour downstaging to increase rates of breast-conserving surgery.

However, whilst it can downstage primary $\mathrm{ER}+$ breast cancer, with less toxicity than chemotherapy, neoadjuvant endocrine therapy has not been widely adopted because of concerns about de novo (intrinsic) resistance to hormonal manipulation. Progression during neoadjuvant endocrine therapy would in theory put patients at increased risk of disease recurrence following surgery, and reduce rates of downstaging (or even operability) compared to those who undergo surgery upfront. Intrinsic resistance is found across multiple studies in the setting of primary and neoadjuvant endocrine therapy (see Fig. 3) [53]. These studies showed a rate of initial disease progression of up to $30 \%$, which is not comparable to that of neoadjuvant chemotherapy (around 3\%) [54]. Most of these studies used tamoxifen, and response rates would be expected to improve with use of AIs or fulvestrant. However, compared to chemotherapy, recent evidence suggests that giving endocrine therapy in the neoadjuvant setting is associated with lower rates of downstaging and complete response [55]. Use of decision-making tools such as Oncotype Dx has been explored to help choose neoadjuvant therapy for breast cancer. For example, for patients with low or intermediate recurrence risk according to the Oncotype test, a feasibility study has suggested that neoadjuvant endocrine therapy is effective, implying that it is preferable to adjuvant chemotherapy for lower-risk patients [56].

\section{PRIMARY ENDOCRINE THERAPY}

Patients with primary breast cancer should be offered curative surgery irrespective of age and ER status-provided they are fit enough for surgery [57]. However, data from UK clinical practice in the previous decade suggest that up 


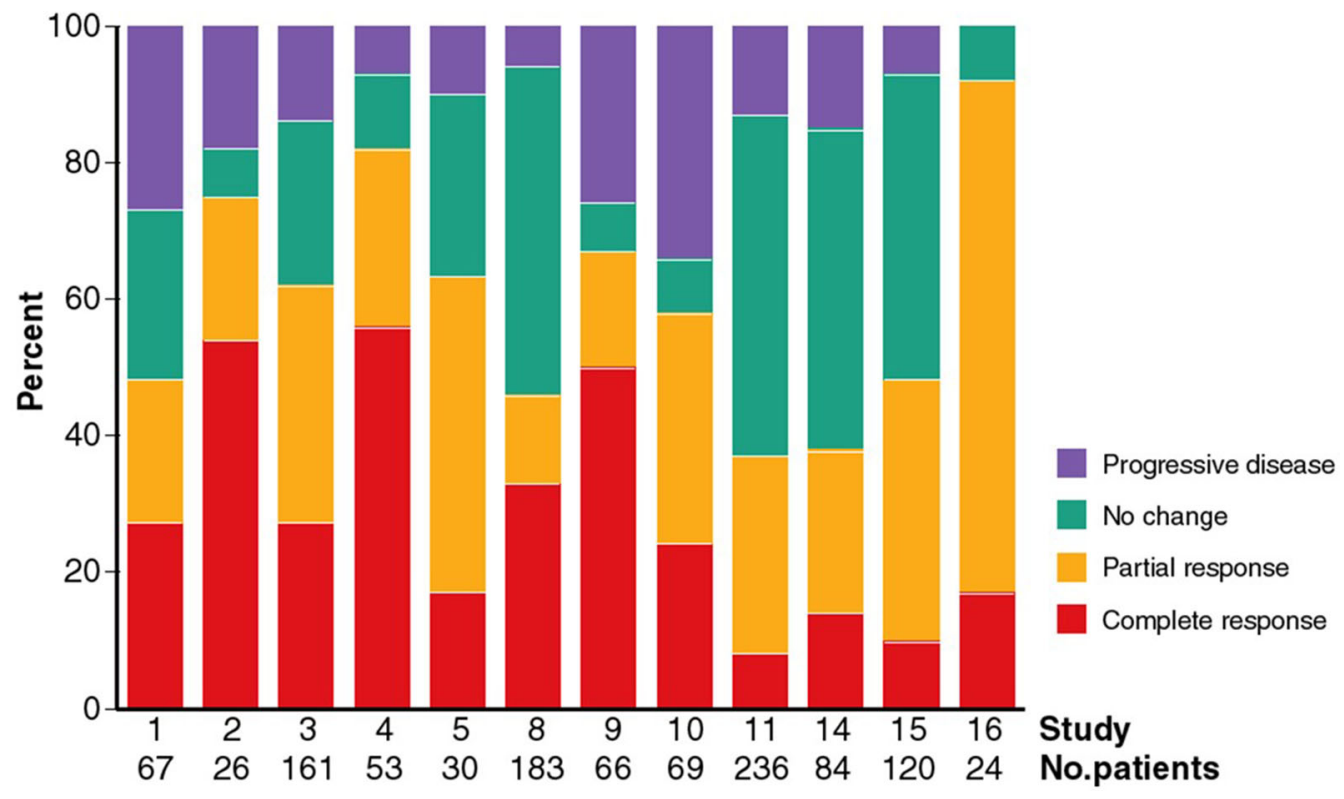

Fig. 3 The primary use of endocrine therapies: response rates in neoadjuvant studies [53]

to $40 \%$ of women over 70 years of age presenting with ER+ disease had primary endocrine therapy (PET) instead of initial surgery [58].

Selection of initial therapy is dictated by patient fitness, co-morbidities and patient choice. Fitness for surgery is assessed clinically, and the decision made is often a factor of complex physical and psychosocial considerations [59].

Not surprisingly, randomised trials of PET versus initial surgery show that removal of the primary tumour (followed by optimal adjuvant therapy) provides better local and regional control than PET [60]. Nearly all patients who have PET will eventually experience progression of the primary tumour due to the development of endocrine resistance. Furthermore, up to $20 \%$ of patients who receive PET will go on to require surgery as a result of disease progression [61].

In 2007, a Cochrane review analysed seven randomised trials of PET versus initial surgery in older women (above 70 years at diagnosis) [62]. All but one of the trials (the Nottingham EPSII study) began before the routine use of ER status to select for endocrine therapy. Despite this, the Cochrane meta-analysis found no overall survival advantage for initial surgery over PET. In 2014, repeated meta-analysis confirmed that these data remain valid at long-term follow-up [60].

At the end of 2007, the ESTEeM study (Endocrine with or without Surgical Therapy for Elderly women with Mammary cancer) was established to provide definitive assessment of PET as a safe and effective breast cancer treatment in older women. Inclusion criteria specified tumours of moderate or strongly positive ER expression by IHC. Unfortunately, the study closed early because of a low rate of patient recruitment to the trial.

In the absence of randomised data from ESTEeM, the effect of selecting patients for PET based on ER status is clearly demonstrated by comparison of the Nottingham Elderly Primary Series randomised trials I and II [61, 63].

In EPSI, patients were not selected for treatment according to ER status. In line with the expected proportion of ER-positive tumours in the patient cohort, $74 \%$ of patients in the EPSI study derived clinical benefit from primary tamoxifen at 6 months. In the EPSII study, patients were selected for PET according to ER status, using ER $H$ score $>100$ as the cut-off for positivity. The rate of clinical benefit correspondingly improved, to $97 \%$ at 6 months. Analysis of a large retrospective clinical cohort 
treated at Nottingham $(N=1078)$ indicates that not only ER status but also the degree of ER positivity is important in selecting patients for PET. In this retrospective cohort, clinical benefit with PET reached $100 \%$ for those patients who had tumours with 'very high' ER positivity (defined as $H$ score $>250$ ) [64] (see Table 4).

The importance of degree of ER positivity is particularly relevant in the context of older women with primary breast cancer. It has been shown that primary breast cancer in older people has biological characteristics distinct from those in younger patients. For example, the proportion of ER-positive disease and degree of ER positivity both increase with age (see Fig. 4) [65]. This suggests that older patients with primary breast cancer are more likely to have ERrich tumours and therefore derive clinical benefit from PET.

The challenge remains to select the right patients for PET using standardised clinical assessment tools to assess frailty, combined with biomarkers in addition to ER status to pick out distinct biological features of those likely to derive benefit from PET [66].

\section{CHEMOPREVENTION}

Chemoprevention in breast cancer uses endocrine therapy to reduce the risk of breast cancer developing in patients considered at high risk. Currently, only patients at high risk are considered for chemoprevention. This is determined by family history and presence of BRCA1/2 and TP53 mutation.
Chemoprevention has also been used for highrisk pathologies including atypical ductal hyperplasia, atypical lobular hyperplasia and lobular carcinoma in situ [67]. An expanded role for endocrine therapy in breast cancer prevention has been explored on the basis of data showing reduced incidence of contralateral breast cancer in large adjuvant endocrine therapy studies.

In the Early Breast Cancer Trialists' Collaborative Group (EBCTCG) meta-analysis of randomised adjuvant trials, 5 years of tamoxifen decreased the rate of contralateral breast cancer from $4.7 \%$ to $2.6 \%$ [68]. This equates to a proportional reduction of $47 \%$, although it should be noted that ER status was not routinely assessed to select patients for adjuvant tamoxifen at the time of all reviewed trials. Subsequent meta-analysis of adjuvant AI trials, all of which did select patients by ER status, revealed a $40 \%$ reduction with AIs at 5 years versus tamoxifen [69], reducing the incidence from $2.0 \%$ to $1.2 \%$ [69].

These preliminary data enabled the hypothesis that chemoprevention would reduce breast cancer incidence to be tested in prospective trials. Similar to therapeutic studies, the concept of balancing efficacy and tolerability (toxicity) is key to chemoprevention strategies.

In terms of efficacy, all the pivotal trials of breast cancer chemoprevention demonstrated a reduction in breast cancer incidence in the experimental arm [70, 71]. For example, in the tamoxifen chemoprevention trials, breast cancer incidence was reduced by $44 \%$ in ER-positive patients [71]. Meta-analysis of trials including

Table 4 Selecting patients for primary endocrine therapy according to degree of ER positivity increases the proportion who have clinical benefit, and reduces the rate of de novo resistance

\begin{tabular}{llll}
\hline All $\geq$ 70 years on primary endocrine therapy (stage 1/2 disease) & Chakrabarti $^{\mathbf{a}}$ & Johnston $^{\mathbf{b}}$ & Syed $^{\mathbf{c}}$ \\
\hline ER $H$ score & Unselected & $H \geq 100$ & $H \geq 250$ \\
$\mathrm{~N}$ & 68 & 100 & 121 \\
$\mathrm{CB}$ (at 6 months) & $74 \%$ & $97 \%$ & $100 \%$ \\
PD (at 6 months) (de novo resistance) & $26 \%$ & $3 \%$ & $0 \%$ \\
\hline a Chakrabarti et al. [63] & & & \\
b Johnston et al. [61] & & \\
c Syed et al. [64] & & \\
\end{tabular}




\section{ER status of primary breast cancer by age}
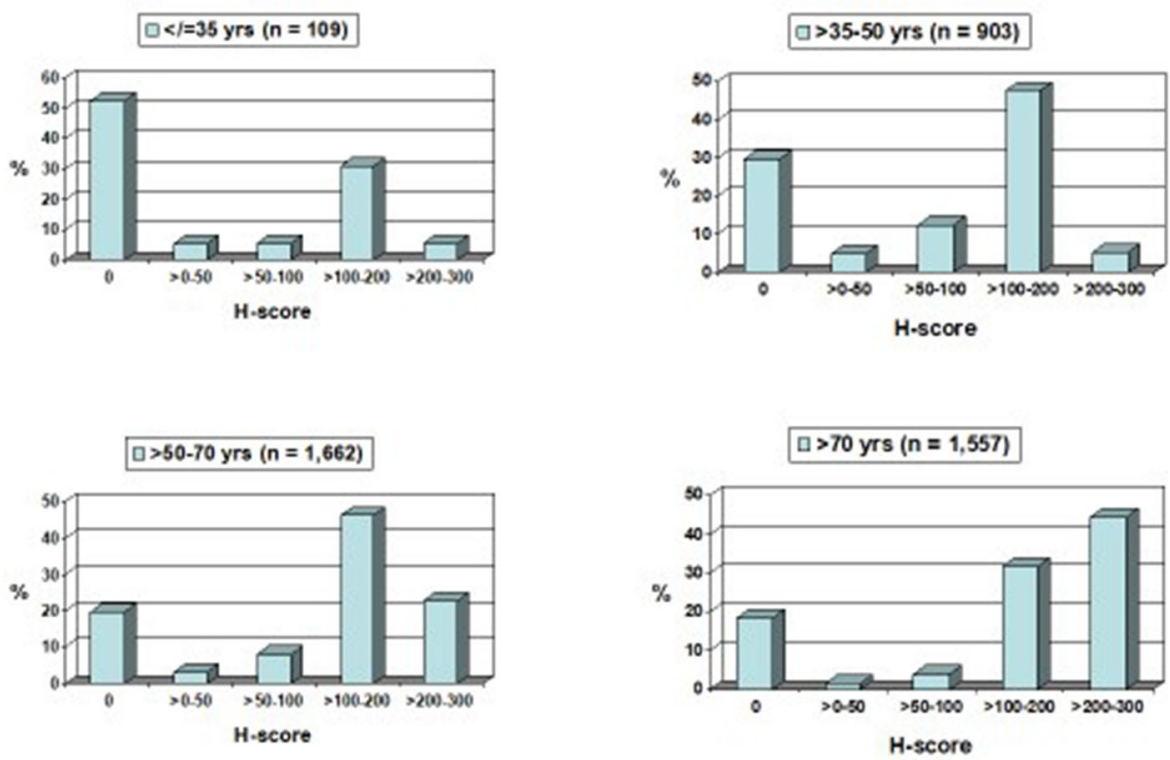

Cheung KL et al, Crit Rev Oncol Hematol 2008

Fig. 4 The relative proportion of ER-positive primary breast cancer increases with age at diagnosis Reprinted from Ref. [65]. Copyright (2008), with permission from Elsevier

AI chemoprevention trials demonstrates a clear benefit for AIs over tamoxifen [70]. This metaanalysis compared relative risk (RR), defined as the ratio between the incidence of breast cancer or serious adverse events in the experimental versus control arm. For AIs, RR was 0.468 [95\% confidence interval (CI) 0.346-0.634] compared to 0.708 (95\% CI $0.595-0.842$ ) for tamoxifen.

This analysis highlights the importance of balancing efficacy with tolerability (toxicity) in chemoprevention. In all studies, the reduction in breast cancer incidence came at the expense of greater incidence of the known complications of endocrine therapies, e.g. loss of bone mineral density on AIs. Serious toxicities on SERMs include thromboembolic events, which were significantly increased across the studies (odds ratio $=1.73, p<0.0001$ ) [71]. In the trials involving SERMs, rate of endometrial cancer was significantly increased (hazard ratio $=1.56$, 1.13-2.14). This effect was seen mainly in the tamoxifen studies, and no effect on endometrial cancer incidence was seen with raloxifene.
Whilst alternative SERMs such as raloxifene have demonstrated favourable benefit-risk ratios over tamoxifen and AIs in chemoprevention studies, they lack data from large adjuvant studies, which may limit their uptake into standard clinical practice.

The role of tamoxifen and AIs in chemoprevention has yet to be fully defined. One outstanding question from the trials is whether this reduction leads consistently to a reduction in overall survival. An alternative hypothesis is that chemoprevention delays but does not prevent breast cancer. Long-term follow-up of the prospective trials is required to resolve this question.

Ultimately, chemoprevention with endocrine therapy will play only part of the role in preventing breast cancer. BRCA-related cancers are predominantly ER negative, hence chemoprevention strategies also involve alternative modalities such as prophylactic mastectomy. Young women at high risk of developing breast cancer are most likely to benefit from 
chemoprevention. The challenge remains to define this group so that clinicians can make informed decisions with patients.

\section{CONCLUSIONS AND FUTURE DIRECTIONS}

Endocrine therapy is the mainstay of treatment of ER-positive breast cancer, with established clinical utility in advanced disease and in the adjuvant and neoadjuvant settings. Rational combination of endocrine therapy with ovarian suppression and newer targeted therapies has maximised efficacy in specific patient subgroups.

Novel therapies have reached the clinic in the first-line advanced setting, and their role is likely to expand into adjuvant and neoadjuvant treatment. Inhibitors of mTOR and CDK4/6 are currently being explored in the adjuvant setting in combination with endocrine therapy $[72,73]$.

In the older population, who comprise the majority of breast cancer patients, such developments may help redefine the role of primary endocrine therapy, alone or in combination with emerging targeted agents. In younger patients at high risk of developing breast cancer, the role of chemoprevention is likely to expand in small increments to larger groups with welldefined risk factors.

Irrespective of the clinical setting, the detection of endocrine treatment failure or resistance is likely to have a substantial impact on future clinical practice. It has been demonstrated that mutations in ESR1, the gene encoding ER, are acquired secondary to endocrine therapy [74]. Similarly, amplification of CYP19 is a potential resistance mechanism that allows ER+ breast cancer cells to escape aromatase inhibition [75]. Detection of these genetic aberrations in serum has the potential to detect treatment failure or resistance at an earlier stage and influence clinical decisionmaking.

There is a multiplicity of therapeutic strategies for ER-positive breast cancer, especially in the advanced setting, where patients are likely to have received prior AI or tamoxifen as part of adjuvant treatment. Despite advances in our understanding of resistance mechanisms and the introduction of novel targeted agents, the only biomarker to select patients for endocrine therapy is expression of the driving transcription factor, ER. The challenge for clinicians is to interpret the available data and select the best strategy for ER-targeted therapy for individual patients.

\section{ACKNOWLEDGEMENTS}

Funding. No funding or sponsorship was received for this study or publication of this article.

Authorship. Both named authors meet the International Committee of Medical Journal Editors (ICMJE) criteria for authorship for this article, take responsibility for the integrity of the work as a whole, and have given their approval for this version to be published.

Disclosures. Simon J. Johnston and KwokLeung Cheung have nothing to disclose.

Compliance with Ethics Guidelines. This article is based on previously conducted studies, some of which were performed by the authors in full compliance with ethics guidelines. The article does not contain any studies with animals performed by either of the authors.

Data Availability. The datasets generated and/or analysed during the current study are available from the corresponding author on reasonable request.

Open Access. This article is distributed under the terms of the Creative Commons Attribution-NonCommercial 4.0 International License (http://creativecommons.org/licenses/ by-nc/4.0/), which permits any noncommercial use, distribution, and reproduction in any medium, provided you give appropriate credit to the original author(s) and the source, provide a link to the Creative Commons license, and indicate if changes were made. 


\section{REFERENCES}

1. Early Breast Cancer Trialists' Collaborative Group. Tamoxifen for early breast cancer: an overview of the randomised trials. Lancet. 1998;351(9114):1451-67.

2. Sommer S, Fuqua SA. Estrogen receptor and breast cancer. Semin Cancer Biol. 2001;11(5):339-52.

3. Beatson GT. On the Treatment of inoperable cases of carcinoma of the mamma: suggestions for a new method of treatment, with illustrative cases. Trans Med Chir Soc Edinb. 1896;15:153-79.

4. Jensen EV. On the mechanism of estrogen action. Perspect Biol Med. 1962;6:47-59.

5. Jordan VC. Molecular mechanisms of antiestrogen action in breast cancer. Breast Cancer Res Treat. 1994;31(1):41-52.

6. Clemons $\mathrm{M}$, Danson S, Howell A. Tamoxifen ("Nolvadex"): a review. Cancer Treat Rev. 2002;28(4):165-80.

7. Fisher B, et al. endometrial cancer in tamoxifentreated breast-cancer patients-findings from the National Surgical Adjuvant Breast and Bowel Project (Nsabp) B-14. J Natl Cancer Inst. 1994;86(7):527-37.

8. Gradishar W. Landmark trials in endocrine adjuvant therapy for breast carcinoma. Cancer. 2006;106(5):975-81.

9. Miller WR. Aromatase inhibitors: mechanism of action and role in the treatment of breast cancer. Semin Oncol. 2003;30(4 Suppl 14):3-11.

10. Lonning PE, et al. Activity of exemestane in metastatic breast cancer after failure of nonsteroidal aromatase inhibitors: a phase II trial. J Clin Oncol. 2000;18(11):2234-44.

11. Cuzick J, et al. Effect of anastrozole and tamoxifen as adjuvant treatment for early-stage breast cancer: 10-year analysis of the ATAC trial. Lancet Oncol. 2010;11(12):1135-41.

12. Johnston SJ, Cheung KL. Fulvestrant - a novel endocrine therapy for breast cancer. Curr Med Chem. 2010;17(10):902-14.

13. Robertson JF, et al. A good drug made better: the fulvestrant dose-response story. Clin Breast Cancer. 2014;14(6):381-9.

14. Robertson JF, et al. Fulvestrant versus anastrozole for the treatment of advanced breast carcinoma in postmenopausal women: a prospective combined analysis of two multicenter trials. Cancer. 2003;98(2):229-38.

15. Agrawal A, et al. Bone turnover markers in postmenopausal breast cancer treated with fulvestrant-a pilot study. Breast. 2009;18(3):204-7.

16. Baselga $\mathrm{J}$, et al. Everolimus in postmenopausal hormone-receptor-positive advanced breast cancer. N Engl J Med. 2012;366(6):520-9.

17. Ciruelos Gil EM. Targeting the PI3K/AKT/mTOR pathway in estrogen receptor-positive breast cancer. Cancer Treat Rev. 2014;40(7):862-71.

18. Spring LM, et al. Clinical management of potential toxicities and drug interactions related to cyclindependent kinase 4/6 inhibitors in breast cancer: practical considerations and recommendations. Oncologist. 2017;22(9):1039-48.

19. Robertson JF, et al. The clinical relevance of static disease (no change) category for 6 months on endocrine therapy in patients with breast cancer. Eur J Cancer. 1997;33(11):1774-9.

20. Forward DP, et al. Clinical and endocrine data for goserelin plus anastrozole as second-line endocrine therapy for premenopausal advanced breast cancer. Br J Cancer. 2004;90(3):590-4.

21. Bonneterre J, et al. Anastrozole versus tamoxifen as first-line therapy for advanced breast cancer in 668 postmenopausal women: results of the Tamoxifen or Arimidex Randomized Group Efficacy and Tolerability study. J Clin Oncol. 2000;18(22):3748-57.

22. Mouridsen $\mathrm{H}$, et al. Phase III study of letrozole versus tamoxifen as first-line therapy of advanced breast cancer in postmenopausal women: analysis of survival and update of efficacy from the International Letrozole Breast Cancer Group. J Clin Oncol. 2003;21(11):2101-9.

23. Paridaens RJ, et al. Phase III study comparing exemestane with tamoxifen as first-line hormonal treatment of metastatic breast cancer in postmenopausal women: the European Organisation for Research and Treatment of Cancer Breast Cancer Cooperative Group. J Clin Oncol. 2008;26(30):4883-90.

24. Nabholtz JM, et al. Anastrozole is superior to tamoxifen as first-line therapy for advanced breast cancer in postmenopausal women: results of a North American multicenter randomized trial. Arimidex Study Group. J Clin Oncol. 2000;18(22):3758-67.

25. Finn RS, et al. Palbociclib and letrozole in advanced breast cancer. N Engl J Med. 2016;375(20):1925-36. 
26. Yau THL, Cheung KL. Optimising endocrine therapy in postmenopausal women with advanced breast cancer. Endocr Relat Cancer. 2018;25(7):705-21.

27. Hortobagyi GN, et al. Ribociclib as first-line therapy for HR-positive, advanced breast cancer. N Engl J Med. 2016;375(18):1738-48.

28. Goetz MP, et al. MONARCH 3: abemaciclib as initial therapy for advanced breast cancer. J Clin Oncol. 2017;35(32):3638-46.

29. Di Leo A, et al. Results of the CONFIRM phase III trial comparing fulvestrant $250 \mathrm{mg}$ with fulvestrant $500 \mathrm{mg}$ in postmenopausal women with estrogen receptor-positive advanced breast cancer. J Clin Oncol. 2010;28(30):4594-600.

30. Robertson JF, et al. Activity of fulvestrant $500 \mathrm{mg}$ versus anastrozole $1 \mathrm{mg}$ as first-line treatment for advanced breast cancer: results from the FIRST study. J Clin Oncol. 2009;27(27):4530-5.

31. Robertson JFR, et al. Fulvestrant $500 \mathrm{mg}$ versus anastrozole $1 \mathrm{mg}$ for hormone receptor-positive advanced breast cancer (FALCON): an international, randomised, double-blind, phase 3 trial. Lancet. 2016;388(10063):2997-3005.

32. Cristofanilli $\mathrm{M}$, et al. Fulvestrant plus palbociclib versus fulvestrant plus placebo for treatment of hormone-receptor-positive, HER2-negative metastatic breast cancer that progressed on previous endocrine therapy (PALOMA-3): final analysis of the multicentre, double-blind, phase 3 randomised controlled trial. Lancet Oncol. 2016;17(4):425-39.

33. Johnston SR, et al. Fulvestrant plus anastrozole or placebo versus exemestane alone after progression on non-steroidal aromatase inhibitors in postmenopausal patients with hormone-receptor-positive locally advanced or metastatic breast cancer (SoFEA): a composite, multicentre, phase 3 randomised trial. Lancet Oncol. 2013;14(10):989-98.

34. Bergh J, et al. FACT: an open-label randomized phase III study of fulvestrant and anastrozole in combination compared with anastrozole alone as first-line therapy for patients with receptor-positive postmenopausal breast cancer. J Clin Oncol. 2012;30(16):1919-25.

35. Mehta RS, et al. Combination anastrozole and fulvestrant in metastatic breast cancer. $\mathrm{N}$ Engl J Med. 2012;367(5):435-44.

36. Kaufman B, et al. Trastuzumab plus anastrozole versus anastrozole alone for the treatment of postmenopausal women with human epidermal growth factor receptor 2-positive, hormone receptor-positive metastatic breast cancer: results from the randomized phase III TAnDEM study. J Clin Oncol. 2009;27(33):5529-37.

37. Ravdin PM, et al. Computer program to assist in making decisions about adjuvant therapy for women with early breast cancer. J Clin Oncol. 2001;19(4):980-91.

38. Wishart GC, et al. PREDICT: a new UK prognostic model that predicts survival following surgery for invasive breast cancer. Breast Cancer Res. 2010;12(1):R1.

39. Sparano JA, et al. Prospective validation of a 21-gene expression assay in breast cancer. N Engl J Med. 2015;373(21):2005-14.

40. Jonat W, et al. Goserelin versus cyclophosphamide, methotrexate, and fluorouracil as adjuvant therapy in premenopausal patients with node-positive breast cancer: the Zoladex Early Breast Cancer Research Association Study. J Clin Oncol. 2002;20(24):4628-35.

41. Francis PA, Regan MM, Fleming GF. Adjuvant ovarian suppression in premenopausal breast cancer. N Engl J Med. 2015;372(17):1673.

42. Ingle JN. Overview of adjuvant trials of aromatase inhibitors in early breast cancer. Steroids. 2011;76(8):765-7.

43. Hind D, et al. Hormonal therapies for early breast cancer: systematic review and economic evaluation. Health Technol Assess. 2007;11(26):1-134 (iii-iv, ix-xi).

44. Goss PE, et al. Extending aromatase-inhibitor adjuvant therapy to 10 years. $\mathrm{N}$ Engl J Med. 2016;375(3):209-19.

45. Davies C, et al. Long-term effects of continuing adjuvant tamoxifen to 10 years versus stopping at 5 years after diagnosis of oestrogen receptor-positive breast cancer: aTLAS, a randomised trial. Lancet. 2013;381(9869):805-16.

46. Gray RG, et al. aTTom: long-term effects of continuing adjuvant tamoxifen to 10 years versus stopping at 5 years in 6953 women with early breast cancer. J Clin Oncol. 2013;31(18_suppl):5. https:// doi.org/10.1200/jco.2013.31.18_suppl.5.

47. Fisher B, et al. Surgical adjuvant chemotherapy in cancer of the breast: results of a decade of cooperative investigation. Ann Surg. 1968;168(3):337-56.

48. Fisher B, et al. Effect of preoperative chemotherapy on the outcome of women with operable breast cancer. J Clin Oncol. 1998;16(8):2672-85. 
49. Eiermann W, et al. Preoperative treatment of postmenopausal breast cancer patients with letrozole: a randomized double-blind multicenter study. Ann Oncol. 2001;12(11):1527-32.

50. Smith IE, et al. Neoadjuvant treatment of postmenopausal breast cancer with anastrozole, tamoxifen, or both in combination: the Immediate Preoperative Anastrozole, Tamoxifen, or Combined with Tamoxifen (IMPACT) multicenter doubleblind randomized trial. J Clin Oncol. 2005;23(22):5108-16.

51. Cataliotti L, et al. Comparison of anastrozole versus tamoxifen as preoperative therapy in postmenopausal women with hormone receptor-positive breast cancer-the Pre-Operative "Arimidex" Compared to Tamoxilen (PROAC7) trial. Cancer. 2006;106(10):2095-103.

52. Kuter I, et al. Dose-dependent change in biomarkers during neoadjuvant endocrine therapy with fulvestrant: results from NEWEST, a randomized Phase II study. Breast Cancer Res Treat. 2012;133(1):237-46.

53. Cheung KL, Howell A, Robertson JFR. Preoperative endocrine therapy for breast cancer. Endocr Relat Cancer. 2000;7(3):131-41.

54. Caudle AS, et al. Impact of progression during neoadjuvant chemotherapy on surgical management of breast cancer. Ann Surg Oncol. 2011;18(4):932-8.

55. LeVasseur N, Yip W, Li H, et al. Comparing outcomes of neoadjuvant endocrine therapy versus chemotherapy in ER-positive breast cancer: Results from a prospective institutional database. J Clin Oncol. 2017;35(15_suppl):581. https://doi.org/10. 1200/JCO.2017.35.15_suppl.581

56. Bear HD, et al. Using the 21-gene assay from core needle biopsies to choose neoadjuvant therapy for breast cancer: a multicenter trial. J Surg Oncol. 2017;115(8):917-23.

57. Hind D, Wyld L, Reed MW. Surgery, with or without tamoxifen, vs tamoxifen alone for older women with operable breast cancer: Cochrane review. Br J Cancer. 2007;96(7):1025-9.

58. Wyld L, et al. Stage and treatment variation with age in postmenopausal women with breast cancer: compliance with guidelines. $\mathrm{Br} \mathrm{J}$ Cancer. 2004;90(8):1486-91.

59. Johnston SJ, Cheung KL. The role of primary endocrine therapy in older women with operable breast cancer. Future Oncol. 2015;11(10):1555-65.
60. Morgan JL, Reed MW, Wyld L. Primary endocrine therapy as a treatment for older women with operable breast cancer-a comparison of randomised controlled trial and cohort study findings. Eur J Surg Oncol. 2014;40(6):676-84.

61. Johnston SJ, et al. A randomised trial of primary tamoxifen versus mastectomy plus adjuvant tamoxifen in fit elderly women with invasive breast carcinoma of high oestrogen receptor content: long-term results at 20 years of follow-up. Ann Oncol. 2012;23(9):2296-300.

62. Hind D, et al. Surgery versus primary endocrine therapy for operable primary breast cancer in elderly women (70 years plus). Cochrane Database Syst Rev. 2006;1:CD004272.

63. Chakrabarti J, et al. A randomised trial of mastectomy only versus tamoxifen for treating elderly patients with operable primary breast cancer-final results at 20-year follow-up. Crit Rev Oncol Hematol. $2011 ; 78(3): 260-4$.

64. Syed BM, et al. Long-term clinical outcome of oestrogen receptor-positive operable primary breast cancer in older women: a large series from a single centre. Br J Cancer. 2011;104(9):1393-400.

65. Cheung KL, et al. Pathological features of primary breast cancer in the elderly based on needle core biopsies-a large series from a single centre. Crit Rev Oncol Hematol. 2008;67(3):263-7.

66. Syed BM, et al. Biology of primary breast cancer in older women treated by surgery: with correlation with long-term clinical outcome and comparison with their younger counterparts. $\mathrm{Br} \mathrm{J}$ Cancer. 2013;108(5):1042-51.

67. Clauser P, et al. Management of atypical lobular hyperplasia, atypical ductal hyperplasia, and lobular carcinoma in situ. Expert Rev Anticancer Ther. 2016;16(3):335-46.

68. Early Breast Cancer Trialists' Collaborative Group. Tamoxifen for early breast cancer: an overview of the randomised trials. Lancet. 1998;351(9114):1451-67.

69. Dowsett $M$, et al. Meta-analysis of breast cancer outcomes in adjuvant trials of aromatase inhibitors versus tamoxifen. J Clin Oncol. 2010;28(3):509-18.

70. Mocellin S, et al. Breast cancer chemoprevention: a network meta-analysis of randomized controlled trials. J Natl Cancer Inst. 2016;108(2):djv318.

71. Cuzick J, et al. Selective oestrogen receptor modulators in prevention of breast cancer: an updated meta-analysis of individual participant data. Lancet. 2013;381(9880):1827-34. 
72. Chavez-MacGregor M, et al. Phase III randomized, placebo-controlled clinical trial evaluating the use of adjuvant endocrine therapy \pm one year of everolimus in patients with high-risk, hormone receptor (HR) positive and HER2-negative breast cancer (BC): SWOG/NRG/Alliance S1207 (NCT01674140). J Clin Oncol. 2015;33(15_suppl). https://doi.org/ 10.1200/jco.2015.33.15_suppl.tps637

73. Mayer E, DeMichele A, Dubsky P, et al. PALLAS: PAlbociclib Collaborative Adjuvant Study: a randomized phase 3 trial of palbociclib with adjuvant endocrine therapy versus endocrine therapy alone for HR +/HER2- early breast cancer. Cancer Res. 2016;76(4 Suppl):OT1-03-21. https://doi.org/10. 1158/1538-7445.SABCS15-OT1-03-21

74. Robinson DR, et al. Activating ESR1 mutations in hormone-resistant metastatic breast cancer. Nat Genet. 2013;45(12):1446-51.

75. Magnani L, et al. Acquired CYP19A1 amplification is an early specific mechanism of aromatase inhibitor resistance in ERalpha metastatic breast cancer. Nat Genet. 2017;49(3):444-50. 